\begin{tabular}{|c|c|}
\hline Title & Photoelectrochemical Properties of a GaP Electrode with an $n / p$ Junction \\
\hline Author(s) & Carlsson, Per; Uosaki, Kohei; Holmström, B.; Kita, Hideaki \\
\hline Citation & $\begin{array}{l}\text { Journal of The Electrochemical Society, 136(2), 524-528 } \\
\text { https://doi.org/10.1149/1.2096674 }\end{array}$ \\
\hline Issue Date & 1989 \\
\hline Doc URL & http:/hdl.handle.net/2115/50252 \\
\hline Rights & $\begin{array}{l}\text { (1) The Electrochemical Society, Inc. 1989. All rights reserved. Except as provided under U.S. copyright law, this work } \\
\text { may not be reproduced, resold, distributed, or modified without the express permission of The Electrochemical Society } \\
\text { (ECS). The archival version of this work was published in J. Electrochem. Soc. } 1989 \text { volume 136, issue 2, 524-528. }\end{array}$ \\
\hline Type & article \\
\hline File Information & JES136-2_524-528.pdf \\
\hline
\end{tabular}

Instructions for use 


\title{
Photoelectrochemical Properties of a GaP Electrode with an $\mathrm{n} / \mathrm{p}$ Junction
}

\author{
Per Carlsson, ${ }^{1}$ Kohei Uosaki, ${ }^{*}$ B. Holmström, ${ }^{1, *}$ and Hideaki Kita \\ Department of Chemistry, Faculty of Science, Hokkaido University, Sapporo 060, Japan
}

\begin{abstract}
The photoelectrochemical (PEC) properties of a GaP electrode containing $\mathrm{p} / \mathrm{n}$ junction have been studied in $0.1 \mathrm{M}$ $\mathrm{H}_{2} \mathrm{SO}_{4}$ and $0.1 \mathrm{M} \mathrm{NaOH}$. This electrode material exemplifies the problem with semiconductor overlayers and how PEC techniques can be used to probe the interior properties of multijunction materials. This system showed both cathodic and anodic photoresponse. If illuminated at $500 \mathrm{~nm}$ and biased more positive than $-0.95 \mathrm{~V} v \mathrm{~s}$. Ag/AgCl, the photocurrent was anodic, but it became cathodic if biased negative of this potential. If the wavelength was $430 \mathrm{~nm}$ the photoanodic response vanished and only photocathodic response was observed. Charge accumulation at the $\mathrm{p} / \mathrm{n}$ and p/electrolyte junctions affects the magnitude of photocurrents and was studied with light modulated photocurrent voltammetry and impedance measurement. From these results we concluded that the magnitude of the photocurrents is determined by the hole transport through the $\mathrm{n} / \mathrm{p}$ and p/electrolyte junctions. The quantum efficiency $(\mathrm{QE})$ increased at $550 \mathrm{~nm}$ and extended to $570 \mathrm{~nm}$ which is not expected for GaP as the bandgap of GaP corresponds to $550 \mathrm{~nm}$. SIMS analysis showed an unexpectedly high concentration of boron in the n-GaP substrate and the epitaxial n-layer and a boron enrichment at the n/substrate interphase. The formation of boron phosphide, which has a bandgap of $\sim 620 \mathrm{~nm}$, during the fabrication process was considered to be responsible for the increased $\mathrm{QE}$ at $550 \mathrm{~nm}$.
\end{abstract}

Photoelectrochemical (PEC) cells have attracted much attention because of their possible use for conversion of solar energy to electricity and/or to chemical energy. High conversion efficiency and the stabiliity of the PEC cells are crucial for any practical application and there are many concepts proposed to improve the stability of PEC cells. The semiconductor electrodes can be stabilized by adding a redox couple to the electrolyte (1), or by covering the electrode surface with a noble metal (2), with a wide bandgap semiconductor (3), or with a conductive polymer (4). However new difficulties appear when electrodes are stabilized by deposition of an overlayer. The efficiency is decreased by light absorption in the overlayer and poor conductivity causes potential drop in the overlayer. If semiconductors are used as an overlayer, problems with band mismatch and junctions appear, and the efficiency is drastically reduced. Multijunction semiconductor materials which contain strained layers, quantum wells, etc., are also of interest for other applications, including ultrahigh speed computer circuits, and are studied also because of their intrinsic scientific interest. As a complement to conventional solid-state techniques, photoelectrochemical (PEC) techniques have been found to be useful for probing the interior of such materials (5). Several reports have been published on "anomalous" photobehavior, i.e., cathodic photoresponse at $n$-type or anodic photoresponse at p-type semiconductors (6). Several explanations such as reduction or oxidation of intermediates state adsorbed on the electrode surface and formation of a $p$ layer near the electrode surface or photoconductive layers in the electrode have been proposed. In this paper we present PEC studies of an $\mathrm{n} / \mathrm{p}$ GaP electrode, which has an internal structure illustrated in Fig. 1. There are two active junctions in this electrode, one at the $n / p$ junction and the other at the $p /$ electrolyte junction. The optical properties of GaP permit us to study the characteristics of these junctions, as GaP has two bandgaps: an indirect bandgap of $2.26 \mathrm{eV}$ (corresponding to $550 \mathrm{~nm})$ and a direct bandgap of $2.72 \mathrm{eV}(455$ $\mathrm{nm}$ ). The direct bandgap absorption coefficient at $2.72 \mathrm{eV}$ is three orders of magnitude larger than the indirect absorption coefficient at $2.26 \mathrm{eV}$ (7). Thus, light with a wavelength under $455 \mathrm{~nm}$ is very efficiently absorbed near the p/electrolyte junction, and the light intensity drops very rapidly towards the interior of the semiconductor. Light between $455-550 \mathrm{~nm}$ penetrates deeper into the semiconductor, and part of it is absorbed in the $n / p$ junction. Therefore, the PEC properties of this electrode is controlled both at the $n / p$ and p/electrolyte junctions. Depending on

*Electrochemical Society Active Member.

'Permanent address: Department of Physical Chemistry, Chalmers/University of Göteborg, S-41296 Göteborg, Sweden. the wavelength of the incident light and the electrode potential, it can exhibit either photocathodic or photoanodic response. Thus, the electrode material of the present study exemplifies some problems encountered with semiconductor overlayers and may serve as a model in understanding such "anomalous" photoelectrochemical behavior.

\section{Experimental}

A GaP n/p junction material prepared on n-GaP (111) substrate by liquid phase epitaxy was obtained from Shinetsu Semiconductor Company, Limited. Doping levels and approximate thickness of the two epitaxial layers determined by the supplier are shown in Fig. 1. Ohmic contacts were made by soldering with In and subsequent heating at $400^{\circ} \mathrm{C}$ for $4 \mathrm{~min}$ in $\mathrm{He}$ atmosphere. The electrode was covered with epoxy resin except for the front side and mounted in a glass tubing.

The electrolyte solutions were either $0.1 \mathrm{M} \mathrm{NaOH}$ or $0.5 \mathrm{M}$ $\mathrm{H}_{2} \mathrm{SO}_{4}$ prepared from reagent grade chemicals and Milli-Q purified water.

Prior to the PEC experiments the electrodes were etched for $2 \mathrm{~min}$ in $\mathrm{HCl}: \mathrm{HNO}_{3}(2: 1)$. The $\mathrm{PEC}$ experiments were carried out in a conventional three-compartment glass cell with a Pyrex window. A Pt foil was used as counterelectrode and $\mathrm{Ag} / \mathrm{AgCl}$ as reference electrode: all potentials are reported with respect to this reference.

The potentiostat was a Hokuto Denko HA-301 or a Nikko Keisoku NPGS-301S. The light source was a 500W xenon lamp (Ushio Electric Company, Limited, UXL-500D-O) with a monochromator (Ritsu Oyo Kogaku, MC-25NP). The light intensity was measured with a light power meter

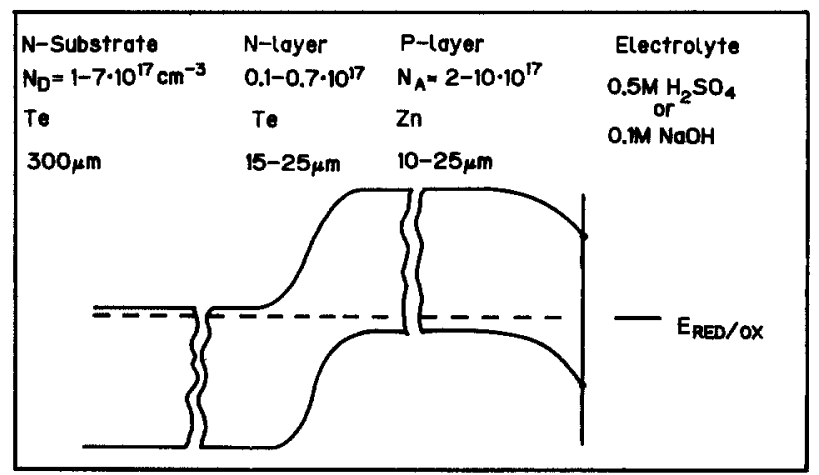

Fig. 1. The schematic band diagram of the $n / p$ GaP electrode at equilibrium. The doping density, doping compound, and approximate thickness of the layers are given. 
(Anritsu Electric Company, Limited, ML-04A with a MA-97A detector), calibrated from 400 to $1100 \mathrm{~nm}$. Quantum conversion efficiency $(\mathrm{QE})$ measurements were controlled and computed with a personal computer (NEC, PC$8801 \mathrm{mkII})$.

The experimental setup for light modulated photocurrent voltammetry is shown in Fig. 2. The electrode was studied under alternating illumination by a xenon lamp with a monochromator, chopped at $80 \mathrm{~Hz}$ (with an NF CH 353 chopper) with or without simultaneous illumination by a xenon lamp with interference filters. The out-of-phase component of the ac photocurrent was measured by using an NF Electronics LT 574 lock-in amplifier as a function of applied potential.

The capacitance measurements were performed with a frequency response analyzer (NF Electronics Instruments, S-5720B) connected to the NEC computer via the GP-IB bus.

A Hitachi U-3200 spectrophotometer was used for absorption measurements. The SIMS measurements were carried out with a CAMECA IMS $3 F$ instrument.

\section{Results}

Voltammetry.-Depending on the bias and on the wavelength of illuminating light, the $n / p$ GaP electrode can exhibit both cathodic and anodic photoresponse, as shown in Fig. 3 for a set of experiments in $0.5 \mathrm{M} \mathrm{H}_{2} \mathrm{SO}_{4}$. When the electrode was illuminated with light of $500 \mathrm{~nm}$, the photocurrent was anodic if the electrode potential was more positive than $-0.95 \mathrm{~V}$, and cathodic if the potential was more negative than this value. If the wavelength of the incident light was $430 \mathrm{~nm}$, the onset of the cathodic photocurrent shifted to $-0.75 \mathrm{~V}$, and there was no anodic photoresponse at positive bias. The anodic photocurrent in $0.5 \mathrm{M} . \mathrm{H}_{2} \mathrm{SO}_{4}$ is due to the photocorrosion of the electrode, and the cathodic photocurrent is due to hydrogen evolution.

Quantum efficiency and spectral response.-The quantum conversion efficiency (QE) action spectra of the $n / p$ GaP electrode at different potentials shown in Fig. 4 further illustrate the general properties seen in the cyclic voltammograms in Fig. 3. The QE was anodic at positive potentials and longer wavelengths, and cathodic at negative potentials and shorter wavelengths. The absolute QE obtained for the $n / p$ GaP electrode was very low, and the $Q E$ shown in Fig. 4 was normalized to the cathodic QE obtained at -1 .3V and $400 \mathrm{~nm}$. The "cross-over wavelength," where the QE changes from anodic to cathodic, and the "onset" wavelength, where the cathodic QE disappeared, were dependent on potential. If the electrode was biased to $-1.3 \mathrm{~V}$, the QE was cathodic at $500 \mathrm{~nm}$ and vanished at 550 $\mathrm{nm}$. At $-0.8 \mathrm{~V}$ the $\mathrm{QE}$ changed from anodic to cathodic at $455 \mathrm{~nm}$.

Figure 4 also shows an increase of the $\mathrm{QE}$ at $550 \mathrm{~nm}$, which is not expected for GaP, since the bandgap wave-

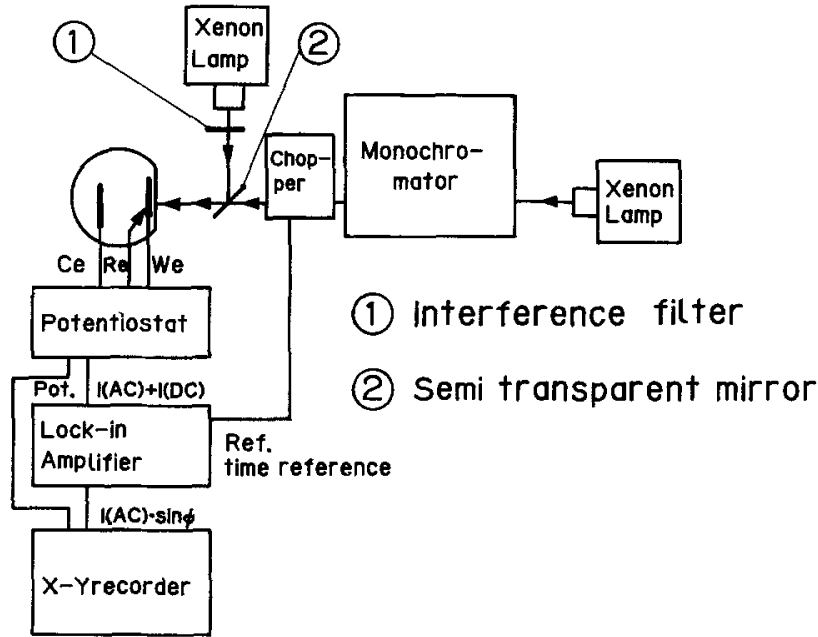

Fig. 2. The experimental block diagram for the light-modulated photocurrent voltammetry measurements.

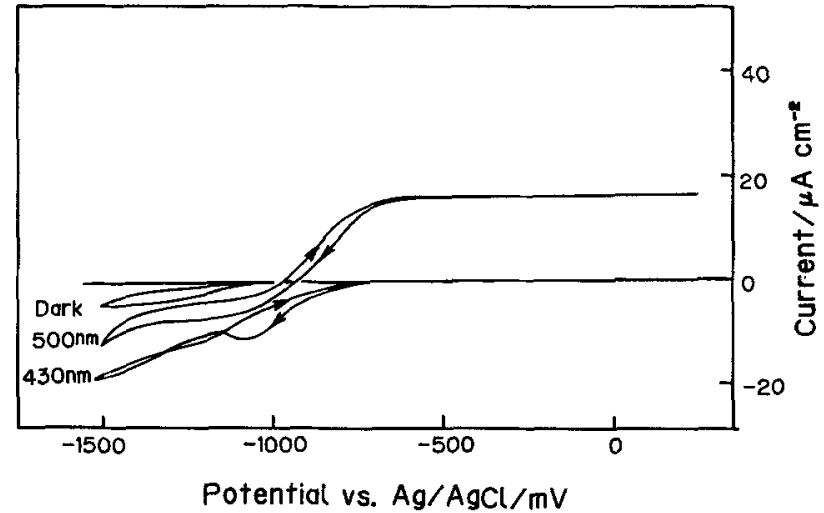

Fig. 3. Cyclic voltammogram of the electrode in $0.5 \mathrm{M} \mathrm{H}_{2} \mathrm{SO}_{4}$ in the dark and under illumination with monochromatic light.

length for the $X$ conduction band is $550 \mathrm{~nm}$. To elucidate this phenomenon further, the differential absorption spectrum between the $n / p$ material and the $n$ substrate material obtained by polishing away the $n / p$ epitaxial layer was recorded between 540 and $600 \mathrm{~nm}$ and is shown as an inset in Fig. 4. The light absorption at $550 \mathrm{~nm}$ was higher in the $\mathrm{n} / \mathrm{p}$ epitaxial material compared to the bulk substrate material.

Light modulated photocurrent (LMP) voltammetry.The upper part of Fig. 5 shows the voltammograms at $\lambda_{\mathrm{AC}}=500 \mathrm{~nm}$ where the principal photoresponse is anodic for three cases: with no dc light and with dc light at 397 and at $553 \mathrm{~nm}$. The L.MP voltammogram obtained without dc illumination was quite different from the ordinary photocurrent voltammogram obtained at the same wavelength ( $c f$. Fig. 3). The anodic photoresponse decreased very slowly at negative potentials, and it did not become photocathodic as in the dc case. If the electrode was simultaneously illuminated with dc light at 397 or $553 \mathrm{~nm}$, the photoanodic response decreases, more for $\lambda_{D C}=553 \mathrm{~nm}$ and less for $\lambda_{\mathrm{DC}}=397 \mathrm{~nm}$.

The lower part of Fig. 5 shows LMP voltammogram obtained at $\lambda_{\mathrm{AC}}=430 \mathrm{~nm}$ where the photoresponse is cathodic. In this case the result without dc illumination was similar to the photocurrent voltammogram. Simultaneous dc illumination at 397 or $553 \mathrm{~nm}$ resulted in a significant increase of the cathodic photoresponse for chopped illumination, larger for $\lambda_{D C}=553$ and less for $397 \mathrm{~nm}$.

Capacitance measurements.-The Mott-Schottky plot of the electrode in the dark (Fig. 6) shows that $1 / C^{2}$ is nearly constant between -2.0 and $-0.1 \mathrm{~V}$ with a weak minimum around $-1.1 \mathrm{~V}$.

Under illumination at $520 \mathrm{~nm}$ the $1 / C^{2}$ decreases, and there is a drastic drop especially at potentials more positive than $-1.0 \mathrm{~V}$, and the minimum now appears at $-0.4 \mathrm{~V}$.

In order to understand the effect of illumination on the capacitance, it was measured at different bias and wave-

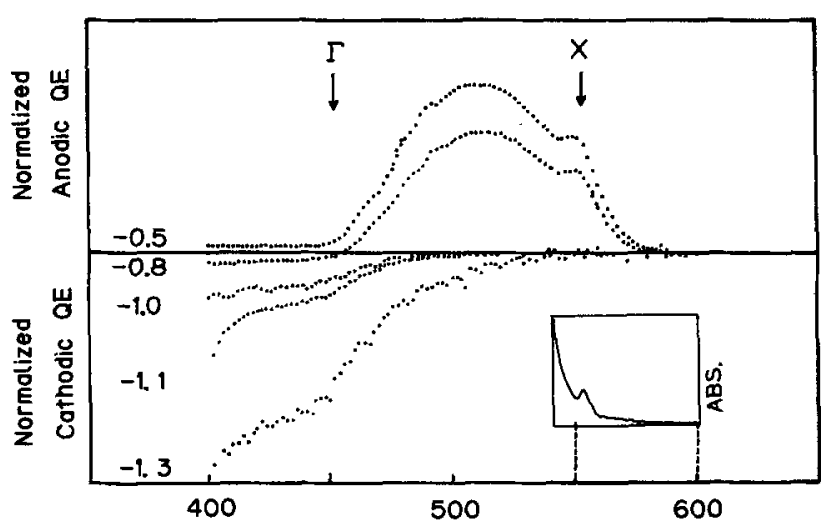

Fig. 4. Normalized light to electricity quantum conversion efficiency (QE) vs. wavelength at five different potentials in $0.5 \mathrm{M} \mathrm{H}_{2} \mathrm{SO}_{4}$. The arrows indicate the wavelength corresponding to the direct $(\Gamma)$ and indirect $(X)$ transitions. Inset: differential absorption spectrum between the $\mathrm{n} / \mathrm{p}$ material and the substrate. 


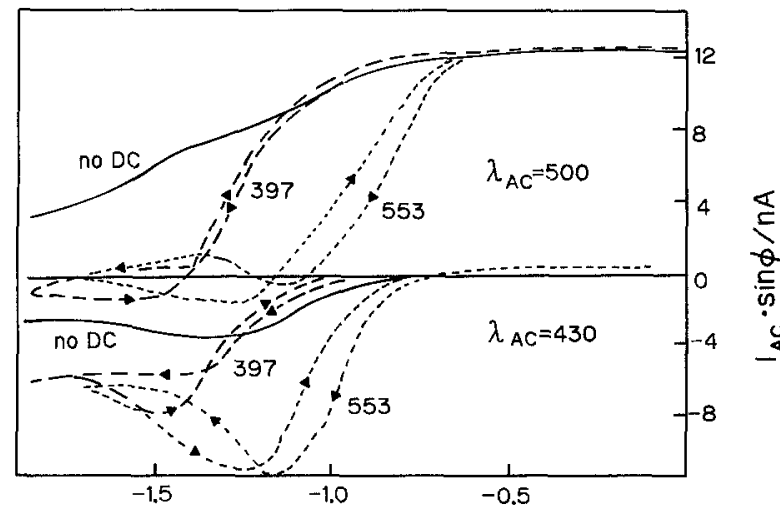

Potential vs. Ag/ $\mathrm{AgCl} / \mathrm{V}$

Fig. 5. The light-modulated photocurrent voltammograms recorded at $\lambda_{\mathrm{AC}}=500 \mathrm{~nm}$ (upper curves) and $\lambda_{\mathrm{AC}}=430 \mathrm{~nm}$ (lower curves) under monochromatic constant illumination at $\lambda_{\mathrm{DC}}=397 \mathrm{~nm}(--)$, at $\lambda_{D C}=553 \mathrm{~nm}(----)$, and without constant illumination (-).

length. The wavelength was varied between 400 and 550 $\mathrm{nm}$ in $10 \mathrm{~nm}$ steps, and the impedance was measured as the potential was changed from -2.2 to $0.2 \mathrm{~V}$ in $0.1 \mathrm{~V}$ steps. Five different frequencies ranging from 20 to $1 \mathrm{kHz}$ were employed for the impedance measurements. The lamp intensity was held constant by adjusting the lamp current and the aperture of the monochromator. The capacitance was evaluated according to an $\mathrm{RC}$ circuit. The assumption of a RC series circuit is only valid in the high frequency region where the contribution from the faradaic component is minimal. The capacitance at $12 \mathrm{kHz}$ is plotted against potential and wavelength, in Fig. 7. The potential-capacitance behavior between 400 and $450 \mathrm{~nm}$ was approximately the same as that observed in the Mott-Schottky plot in the dark. At longer wavelengths, the capacitance increased and reached a maximum at $-1.1 \mathrm{~V}$ and $520 \mathrm{~nm}$. The capacitance was larger between -1.1 and $0 \mathrm{~V}$ at wavelengths over $450 \mathrm{~nm}$ than the capacitance at potentials between -1.1 and $-2.2 \mathrm{~V}$ at corresponding wavelengths. The maximum capacitance was obtained in the same potential and wavelength region where the largest effect of the dc illumination on the light modulated photocurrent voltammogram was obtained (cf. Fig. 5).

pH dependence.-The shift of flatband potential due to the change of $p \mathrm{H}$ of the electrolyte which is usually observed at semiconductor electrodes was also observed at the $\mathrm{n} / \mathrm{p} \mathrm{GaP}$ electrode. The minimum in the Mott-Schottky plot in the dark was shifted by $0.5 \mathrm{~V}$ more negative in $0.1 \mathrm{M}$ $\mathrm{NaOH}$ compared to that in $0.5 \mathrm{M} \mathrm{H}_{2} \mathrm{SO}_{4}$. The crossover po-

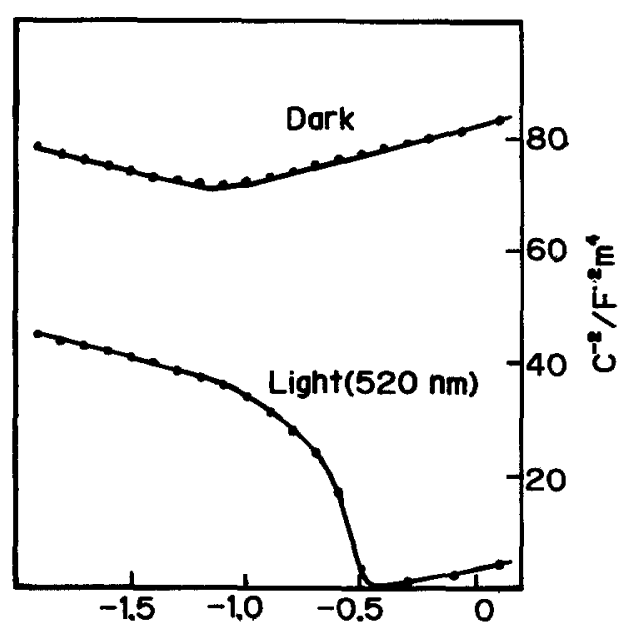

Potential vs. Ag/AgCl/V

Fig. 6. The Mott-Schottky plot of the $n / p$ GoP electrode in $0.5 \mathrm{M}$ $\mathrm{H}_{2} \mathrm{SO}_{4}$ in dark and under illumination with monochromatic light at $520 \mathrm{~nm}$.

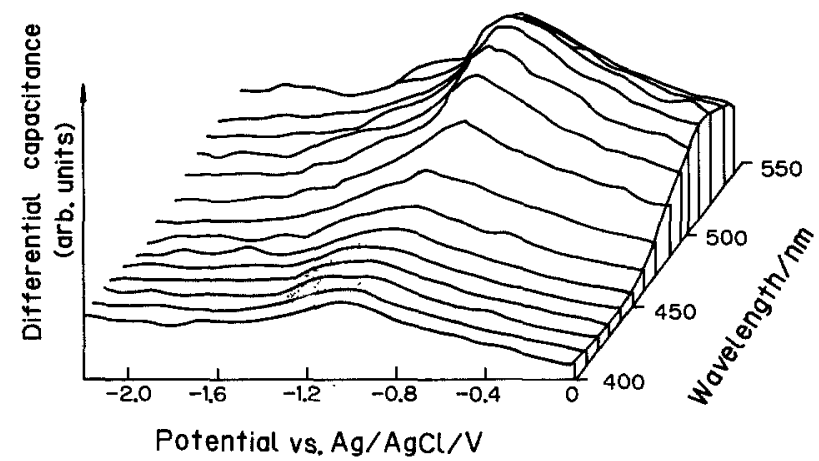

Fig. 7. The differential capacitance of the $\mathrm{n} / \mathrm{p} \mathrm{GaP}$ electrode vs. potential and wavelength in $0.5 \mathrm{M} \mathrm{H}_{2} \mathrm{SO}_{4}$.

tential where the photoresponse changed from photoanodic to photocathodic also shifted by $0.5 \mathrm{~V}$ to more negative potentials. The shift was about $40 \mathrm{mV} / \mathrm{pH}$ which is less than the reported value for $\mathrm{p}-\mathrm{GaP}$ of $50 \sim 59 \mathrm{mV} / \mathrm{pH}(8)$. This is observed in both the ordinary and LMP voltammograms. The photocathodic current decreased in $0.1 M$ $\mathrm{NaOH}$. The $p \mathrm{H}$ dependence of the electrode is consistent with a change of the potential drop at the Helmholtz layer. The whole band diagram shifts toward more negative potential at higher $p \mathrm{H}$.

SIMS analysis.-The electrode material was analyzed with SIMS and the composition-depth profile was recorded to $100 \mu \mathrm{m}$ for the elements and mass numbers as indicated in Fig. 8. The $\mathrm{Ga}_{2}$ and $\mathrm{P}$ signals are almost constant throughout the structure. Unfortunately, the doping compounds, $\mathrm{Zn}$ and Te, were not detectable due to secondary reactions with the sputtering ion $\left(\mathrm{O}^{2-}\right)$ which forms oxidized molecules of $\mathrm{Zn}$ and Te which could not be separated from a variety of Ga-oxygen compounds formed in the sputtering process. Consequently it was not possible to determine the location of the $n / p$ junction.

The most remarkable observation was the boron profile. The concentration of boron was low at the surface and increased slowly in the epitaxial $\mathrm{p} / \mathrm{n}$ layer. The concentration increased by three orders of magnitude at a depth of $\sim 45 \mu \mathrm{m}$ which approximately corresponds to the interface between the epitaxial layer and the substrate material. The boron concentration in the substrate material was constant between 60 and $100 \mu \mathrm{m}$ and was approximately two orders of magnitude higher than that in the epitaxial layer. In an intermediate region between 45 and $60 \mu \mathrm{m}$ the boron signal varied significantly.

\section{Discussion}

Anodic and cathodic photoresponse-As discussed briefly in the introductory section, absorption takes place either in the surface region or in the interior of the semiconductor including the $n / p$ junction, depending on wave-

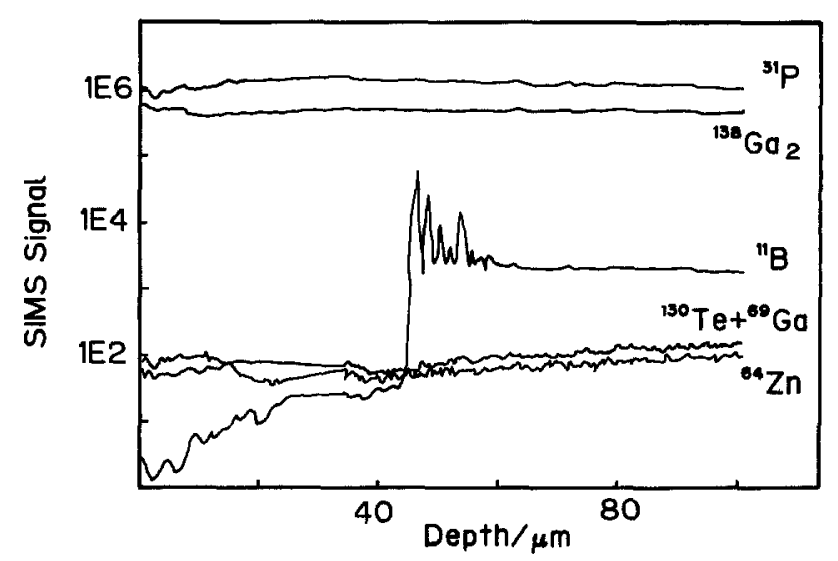

Fig. 8. SIMS profile plot of the $n / p$ GaP material. The relative concentrations for the mass numbers and corresponding elements are plotted vs. depth. 
length of the incident light. The potential and wavelength dependence of the photoresponse can be explained with the band diagrams shown in Fig. 9. The potential and wavelength conditions giving rise to the anodic photocurrent are sketched in Fig. 9a and those giving rise to the cathodic one in Fig. 9b. As shown in Fig. 9a, where the electrode is biased positively, the band bending at the p/electrolyte side decreases and the band bending at the $\mathrm{n} / \mathrm{p}$ junction increases. In this case the photoresponse vanishes if the wavelength is below $450 \mathrm{~nm}$ while the photoresponse above $450 \mathrm{~nm}$ is photoanodic. The light longer than $450 \mathrm{~nm}$ has not sufficient energy to excite the electrons to the $\Gamma$ conduction band, so the electrons are excited only to the $\mathrm{X}$ conduction band. In this case, the light penetrates deeper into the electrode and reaches the $n / p$ junction. For example, while $450 \mathrm{~nm}$ light penetrates only $3 \sim 4 \mu \mathrm{m}$, penetration depth for $500 \mathrm{~nm}$ light is $\sim 30 \mu \mathrm{m}$. The magnitude of this photoanodic current is controlled by the hole transport through the p/electrolyte junction. The electrons are transported to the back contact and the holes are transported through the $\mathrm{p}$ layer to the electrolyte. The hole transport through the $\mathrm{p}$ layer is inhibited at the p/electrolyte junction if the barrier for the holes is too high.

As shown in Fig. 9b, when the electrode is biased negatively, the band bending at the p/electrolyte side increases while the band bending at the $n / p$ junction decreases. If the wavelength of the incident light is $<450 \mathrm{~nm}$, the electrons are excited to the $\Gamma$ conduction band, and the light is absorbed close to the electrolyte side, and the result will be a photocathodic current.

The limiting factor for both the cathodic and anodic photocurrent is the transport of holes. The cathodic photocurrent is limited by the hole transport through the $\mathrm{n} / \mathrm{p}$ junction, and the anodic photocurrent is limited by the hole transport through the p/electrolyte junction. The hole transport through the $\mathrm{n} / \mathrm{p}$ junction could be accomplished by either of two processes: (i) the current is carried through the $n$ region by minority carriers (holes), or (ii) the holes recombine with electrons at the $n / p$ junction, and the current is carried through the $\mathrm{n}$ region by majority carriers (electrons). The increased cathodic ac photocurrent which was observed in the ac voltammogram under illumination with a monochromatic dc light source is attributed to an effect of charge accumulation. If holes accumulate in the $p$

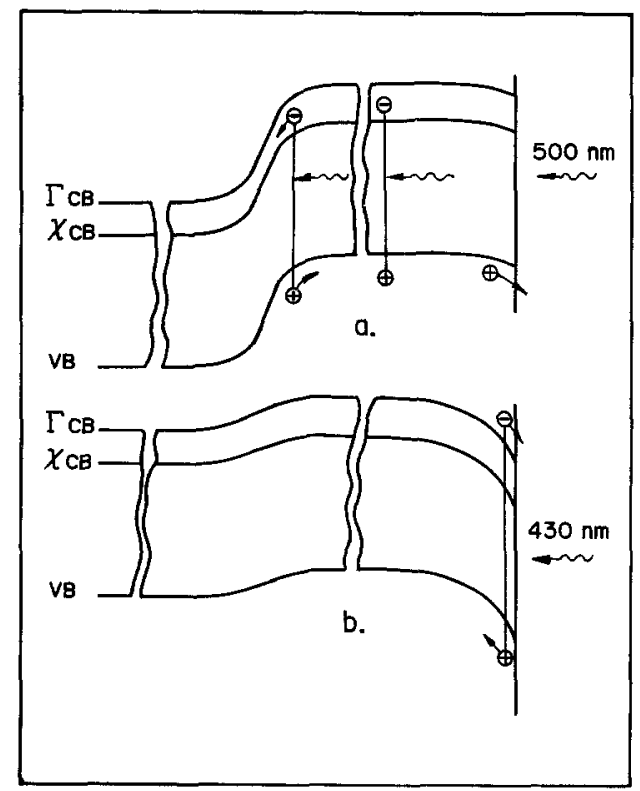

Fig. 9. The schematic band models showing the bias and wavelength condition which generates (a) the photoanodic response and (b) the photocathodic response. In (a) the electrode is anodically biased and illuminated at $500 \mathrm{~nm}$. In this case the $\mathrm{p} / \mathrm{n}$ junction is photoactive. The holes moves towards the electrode surface and the electrons towards the back contact; the net photocurrent is anodic. In (b) the bias is cathodic and the wavelength is $430 \mathrm{~nm}$. In this case the p/electrolyte junction is photoactive. The electrons move towards the electrode surface and the holes to the back contact. region or electrons in the $\mathrm{n}$ region, the band bending over the $n / p$ junction will decrease, and it will be easier for holes to move through the $n / p$ junction. The effect of de illumination was larger for a dc wavelength of $553 \mathrm{~nm}$ than for $397 \mathrm{~nm}$. At $553 \mathrm{~nm}$, the absorbed light excites an electron into the $\mathrm{X}$ conduction band. For this transition, the absorption coefficient and recombination rate are low which are favorable for charge accumulation. Thus, in this case the light penetrates deep into the electrode and reaches the $\mathrm{n} / \mathrm{p}$ junction, and both electrons and holes contribute to the charge accumulation, the holes in the $\mathrm{p}$ region and the electrons in the $n$ region. The dc light at $397 \mathrm{~nm}$ is absorbed close to the electrode surface, and holes contribute less to the charge accumulation, since the recombination rate is high in the direct bandgap and the holes have to be transported to the vicinity of the $n / p$ region. The electrons are transported towards the electrode surface, and they will not contribute to the charge accumulation. This model is also consistent with the reduced anodic ac photoresponse observed under dc illumination. If charge accumulation reduces the band bending at the $n / p$ junction, it would result in less efficient electron-hole pair separation and, thus, a lower anodic ac photocurrent.

It might be that the electron accumulation in the $\mathbf{n}$ region of the $n / p$ junction contributes most to the charge accumulation and the lowering of the barrier height for the hole transport through the lowering of the barrier height for the hole transport through the $\mathrm{n} / \mathrm{p}$ junction.

Capacitance.-The capacitance behavior and the MottSchottky plot of this electrode can be interpreted as two capacitors in series, one corresponding to the $n / p$ junction and the other one to the p/electrolyte junction. The space charge capacitance of each junction, $C_{\mathrm{j}}$, as a function of potential over the junction, is given by Eq. [1] and the series capacitance is given by Eq. [2]

$$
\begin{gathered}
C_{\mathrm{j}}=\left(q N_{\mathrm{sc}} \varepsilon \varepsilon_{\mathrm{o}} / 2\right)^{1 / 2}\left(V_{\mathrm{s}}-k T / q\right)^{-1 / 2} \\
1 / \mathrm{C}=1 / C_{\mathrm{n} / \mathrm{p}}+1 / C_{\mathrm{p} / \mathrm{el}}
\end{gathered}
$$

where $V_{s}$ is the potential over the junction. If the electrode is biased negatively, the capacitance at the $n / p$ junction increases as the potential over this junction decreases, and the total capacitance is, therefore, mainly determined by the p/electrolyte junction. The Mott-Schottky slope is therefore negative at negative potentials. At positive bias, the total capacitance is dominated by the $n / p$ junction and the Mott-Schottky slope is positive in this case. The capacitance behavior during illumination is interpreted as charge accumulation (9) in the interior of the semiconductor. The capacitance-voltage behavior at wavelengths under $450 \mathrm{~nm}$ is similar to that in dark. At wavelengths over $450 \mathrm{~nm}$, the light penetrates into the epitaxial layers and the capacitance increases especially in the potential region around $-1.0 \mathrm{~V}$. In this potential region, it is likely that the capacitance contribution from the $n / p$ junction and the p/electrolyte junction is about the same. If the electrode is illuminated at wavelengths over $450 \mathrm{~nm}$, charge accumulation in the $n$ and $p$ region will change the capacitance especially in this potential region. At negative potentials, the capacitances determined by the p/electrolyte junction and the effect of illumination $>450 \mathrm{~nm}$ is much less, because charge accumulation will not affect the $p /$ electrolyte capacitance. When the electrode is illuminated at wavelengths under $450 \mathrm{~nm}$, the light is absorbed in $\mathrm{p} /$ electrolyte junction but the change in capacitance is very small. The capacitance-wavelength behavior at positive potentials is controlled by the capacitance of $n / p$ junction and the effect of illumination is much more pronounced compared to the situation at negative potentials. At wavelengths over $450 \mathrm{~nm}$ the light penetrates to the $\mathrm{n} / \mathrm{p}$ junction and charge will accumulate in the $n$ and $p$ region and increase the capacitance.

The effect of boron.-The SIMS profile plot reveals the presence of boron in the electrode. Boron belongs to group III and can form a compound with phosphorus, BP, which is a semiconductor with a bandgap of $2.0 \mathrm{eV}$. The SIMS signal of boron between 60 and $100 \mu \mathrm{m}$ is relatively high and constant but is low near the surface. Thus, boron 
seems to originate from the substrate material (10). In an intermediate region between 45 and $55 \mu \mathrm{m}$ the boron signal varies quite significantly and this might be due to inclusions of boron. This depth corresponds approximately to the interface between the substrate and the $\mathrm{n}$ layer. It is therefore likely that boron in the substrate reacts with phosphorus and forms BP in the epitaxial growth process.

The presence of BP in the $n / p$ layer could explain the increased QE at $550 \mathrm{~nm}$. This behavior is not likely for GaP because the $\mathrm{GaP}$ bandgap is $2.26 \mathrm{eV}$ which corresponds to $550 \mathrm{~nm}$. The bandgap of BP corresponds to $620 \mathrm{~nm}$, and therefore $B P$ could account for the increased $Q E$ at $550 \mathrm{~nm}$ and the extension of the photoresponse to $\sim 570 \mathrm{~nm}$ (11). The differential absorption spectrum between the electrode material and the substrate also reveals that the light absorption of these wavelength is higher in the epitaxial layer. It seems that BP plays an important role for the photoelectrochemical behavior of this electrode. The presence of it also complicates the interpretation of the photoelectrochemical properties of this electrode. The band mismatch between GaP and BP could create very efficient traps for electrons or holes, and charge accumulation in these may drastically change the potential barrier at the $n / p$ junction.

\section{Conclusion}

The photoelectrochemical properties of an n/p GaP electrode are determined by the $n / p$ junction and the p/electrolyte junction. The magnitude of the photoanodic and photocathodic currents are controlled by the hole transport through the structure. During illumination we observed charge accumulation in the $\mathrm{p}$ layer which reduced the bandbending and improved the cathodic ac photocurrent. The observation of charge accumulation is also supported by the capacitance measurements. The SIMS profile plot reveals the presence of boron in the epitaxial structure, and the increased $\mathrm{QE}$ at $550 \mathrm{~nm}$ is attributed to light absorption in boron phosphide.

\section{Acknowledgments}

The visits of P.C. and B.H. to Japan were made possible by generous grants from the Swedish Board for Technical Development (STU) and the Japan Society for Promotion of Science, respectively. The $n / p$ junction semiconductor material was a gift from Shin-etsu Semiconductor Company, Limited. The SIMS measurements were performed by Dr. Hans Odelius at the Materials Center, Chalmers/ University of Goteborg.

Manuscript submitted March 3, 1988; revised manuscript received July 25,1988 . This was in part Paper 872 presented at the Honolulu, HI, Meeting of the Society, Oct. 18-23, 1987.

Hokkaido University assisted in meeting the publication costs of this article.

\section{REFERENCES}

1. (a) A. Heller, H. J. Lewerenz, and B. Miller, J. Am. Chem. Soc., 103, 200 (1980); (b) S. Menezes, A. Heller, and B. Miller, This Journal, 127, 1268 (1980).

2. Y. Nakato, K. Abe, and H. Tsubomura, Ber. Bunsenges. Phys. Chem., 80, 1002 (1976).

3. (a) J. O'M. Bockris and K. Uosaki, Energy, 1, 76 (1976); (b) P. A. Kohl, S. N. Frank, and A. J. Bard, This Journal, 124, 225 (1977); (c) D. S. Ginley, R. Y. Baughman, and M. A. Butler, ibid., 130, 1999 (1983).

4. T. Skotheim, I. Lundstrom, and J. Prejza, ibid., 128, 1625 (1981).

5. (a) B. Holmström, P. Carlsson, and L. Samuelson, in "Proceedings of the 5th International Symposium on Photochemical Conversion and Storage of Solar Energy," Osaka, 1984; (b) A. J. Nozik, B. R. Thacker, J. A. Turner, J. Klem, and H. Morkoc, Appl. Phys. Lett., 50, 34 (1987).

6. (a) R. Yoneda, K. Uosaki, and H. Kita, Submitted to This Journal; (b) R. H. Wilson, ibid., 127, 228 (1980); (c) S.-E. Lindquist and H. Vidarsson, J. Mol. Cat., 38, 131 (1986); (d) H. Morisaki, M. Hariya, and K. Yazawa, Appl. Phys. Lett., 30, 7 (1977); (e) N. Muller, G. Hodes, and B. Vainos, J. Electroanal. Chem., 179, 155 (1984); (f) R. Tenne and W. Giriat, ibid., 186, 127 (1985).

7. "Handbook of Optical Constants of Solids," E. D. Palik, Editor, Academic Press, Inc., London (1985).

8. (a) S. R. Morrison, in "Electrochemistry at Semiconductor and Oxidized Metal Electrodes," p. 184, Plenum Press, New York (1980); (b) G. Horowitz, ibid. 49, 357 (1978); (c) M. A. Butler and D. S. Ginley, This Journal, 12\%, 1273 (1980).

9. (a) J. Y. Kelly and P. H. L. Notten, ibid., 130, 2452 (1983); (b) D. Vanmaekelbergh, W. P. Gomes, and F. Cardon, ibid., 134, 891 (1987).

10. P. B. Klein, R. E. R. Nordqvist, and P. G. Siebenman, J. Appl. Phys., 51, 4861 (1980).

11. J. Lee, A Fujishima, K. Honda, and Y. Kumashiro, Bull. Chem. Soc. Jpn., 58, 2634 (1985).

\title{
The Effect of Composition and Phase Change of Electrode Material on Thermoelectric Power in Molten Salt Systems
}

\author{
Masahiro Kamato, Yasuhiko Ito, ${ }^{*}$ Mikio Inoue, and Jun Oishi \\ Department of Nuclear Engineering, Faculty of Engineering, Kyoto University, Sakyo-ku, Kyoto 606 Japan
}

\section{ABSTRACT}

Thermoelectric power measurements were carried out in $\mathrm{Sn}-\mathrm{Li}$ alloy/LiCl-KCl/Sn-Li alloy systems. The thermoelectric power shows different behavior according to the composition and the phase state of the electrode. This behavior has been explained thermodynamically.

Thermoelectric power can be one of the driving forces for mass transfer in nonisothermal systems, and, on the other hand, the single electrode Peltier heat of electrochemical reactors, such as fuel cells or electrolyzers, can be calculated from the thermoelectric power. The latter is very important in designing the energy balance of reactors and the former in studying corrosion of heat transfer loops.

Though many papers (1-5) have been presented concerning the theoretical and/or experimental study of ther-

\footnotetext{
* Electrochemiçal Society Active Member.
}

moelectric power, almost no report exists that uses an alloy in a single or coexisting phases as an electrode.

From an engineering viewpoint thermoelectric power measurement with an alloy electrode is very important, because the construction material of heat transfer loops is an alloy in most cases (6) and electrodes of electrochemical reactors are not necessarily pure metal (7).

From such a viewpoint, we have studied various aspects of thermoelectric power, especially focusing on how the thermoelectric power is affected by the composition or phase change of the electrode material. 